\title{
Neutrino-cooled Accretion Model with Magnetic Coupling for X-ray Flares in GRBs
}

\author{
Yang Luo, Wei-Min Gu, Tong Liu, and Ju-Fu Lu \\ Department of Astronomy and Institute of Theoretical Physics and Astrophysics, \\ Xiamen University, Xiamen, Fujian 361005, China
}

\begin{abstract}
The neutrino-cooled accretion disk, which was proposed to work as the central engine of gamma-ray bursts, encounters difficulty in interpreting the X-ray flares after the prompt gamma-ray emission. In this paper, the magnetic coupling between the inner disk and the central black hole is taken into consideration. For mass accretion rates around $0.001 \sim 0.1 M_{\odot} \mathrm{s}^{-1}$, our results show that the luminosity of neutrino annihilation can be significantly enhanced due to the coupling effects. As a consequence, after the gamma-ray emission, a remnant disk with mass $M_{\text {disk }} \lesssim 0.5 M_{\odot}$ may power most of the observed X-ray flares with the rest frame duration less than 100 seconds. In addition, a comparison between the magnetic coupling process and the Blandford-Znajek mechanism is shown on the extraction of black hole rotational energy.
\end{abstract}

Subject headings: accretion, accretion disks - black hole physics - gamma-ray burst: general - magnetic fields

\section{Introduction}

The launch of Swift satellite has led to tremendous discoveries of gamma-ray bursts (GRBs) (see Mészáros 2006; Gehrels et al. 2009, for a review). A surprising one is that large X-ray flares are common in GRBs and occur at times well after the initial prompt emission (Romano et al. 2006; Falcone et al. 2007; Chincarini et al. 2007; Bernardini et al. 2011). The $\mathrm{X}$-ray flare is an episodic phenomena showing a sudden brightness at the late afterglow stage. From the spectral and temporal analysis of X-ray flares, it is strongly suggested that X-ray flares may have a common origin as the prompt gamma-ray pulses and are related to the late time activity of the central engine (Bernardini et al. 2011; Romano et al. 2006).

For the energy reservoir of powering GRBs or X-ray flares, it is believed that they are produced through an ultra-relativistic jet, i.e., a neutrino annihilation-driven jet or a 
Poynting flux-dominated jet. For the former jet, neutrinos annihilate above the disk and form a hot fireball which subsequently expand to accelerate the jet by its thermal pressure (Popham et al. 1999; Di Matteo et al. 2002; Chen \& Beloborodov 2007). On the other hand, in the case of a Poynting flux-dominated jet, the jet derives its energy from the rotational energy of the central black hole (BH) via the Blandford-Znajek (BZ) process (Blandford \& Znajek 1977; McKinney \& Gammie 2004; Tchekhovskoy et al. 2008). Several mechanisms were proposed to explain the episodic phenomenon of X-ray flares, including fragmentation of a rapidly rotating core (King et al. 2005), magnetic regulation of the accretion flow (Proga \& Zhang 2006), fragmentation of the accretion disk (Perna et al. 2006), differential rotation in a post-merger millisecond pulsar (Dai et al. 2006), transition from thin to thick disk (Lazzati et al. 2008), He-synthesis-driven winds (Lee et al. 2009), the propagation instabilities in GRB jets (Lazzati et al. 2011), and the episodic, magnetically dominated jets (Yuan \& Zhang 2012). Apart from the energy and the episodic activity of X-ray flares, their evolution has also been investigated, which shows that the average energy released in the form of X-ray flares overlaid on the power-law decay of the afterglow of GRBs (Lazzati et al. 2008; Margutti et al. 2011).

In the present work, we will concentrate on another issue, i.e., whether or not the remnant disk after the prompt gamma-ray emission can power X-ray flares through neutrino annihilation. The luminosity of neutrino annihilation produced by the accretion disk is sensitive to the accretion rate. In the late stage of disk evolution, the accretion rate will probably be quite low (e.g., $\sim 0.01 M_{\odot} \mathrm{s}^{-1}$ ) and the annihilation luminosity will drop sharply according to previous numerical calculations (e.g., Popham et al. 1999). In other words, the observed X-ray flares, if produced by the annihilation mechanism, will still require a relatively high accretion rate. We take the second flare of GRB 070318 as an example, which has an isotropic luminosity of $2.96 \times 10^{48} \mathrm{erg} \mathrm{s}^{-1}$ and a rest frame duration of $80.6 \mathrm{~s}$ (see Table 1). In order to produce such a luminosity by neutrino annihilation, an accretion rate around $0.06 M_{\odot} \mathrm{s}^{-1}$ is required for a rapidly rotating $\mathrm{BH}\left(a_{*}=0.9\right)$ due to our numerical calculations in Section 3, which is consistent with the results in Popham et al. (1999). Subsequently, the required remnant disk mass is around $4.8 M_{\odot}$, which is obviously beyond what the progenitor can provide (e.g., Shibata \& Taniguchi 2008; Pannarale et al. 2011).

In this work, we will investigate the neutrino-cooled disk with the magnetic coupling (MC) between the inner disk and the $\mathrm{BH}$, where the magnetic field connects the accretion disk and the central BH (Li 2002; Wang et al. 2002; Uzdensky 2005). In such a model, the angular momentum and the energy are transported from the $\mathrm{BH}$ horizon to the accretion disk through a closed magnetic field. Accordingly, this transported energy will be released and therefore increase the radiation of the disk (Li 2002). The MC effects on the structure and radiation of neutrino-cooled disks have first been studied by Lei et al. (2009). They showed that both 
the neutrino luminosity and the annihilation luminosity will increase significantly owing to the MC process. In the present work, we will focus on relatively low accretion rates $0.001 \sim$ $0.1 M_{\odot} \mathrm{s}^{-1}$ to study the possibility of the remnant disk to power X-ray flares. We would point out that, although such type of magnetic fields is one of the possible field geometries discussed by McKinney (2005, Figure 2) and has been studied by a few previous works, the existence of such magnetic fields remains a problem according to MHD simulations. We will discuss this issue in the last section.

The paper is organized as follows. Equations are presented in Section 2. The structure and neutrino radiation of the disk are calculated in Section 3. A comparison of our numerical results with the observed X-ray flares is shown in Section 4. Conclusions and discussion are made in Section 5.

\section{Equations}

For neutrino-dominated accretion, the disk is extremely hot and dense and the neutrino radiation can balance the viscous dissipation. The structure and radiation of such a disk have been widely investigated in previous works (Popham et al. 1999; Di Matteo et al. 2002; Gu et al. 2006; Chen \& Beloborodov 2007; Liu et al. 2007; Lei et al. 2009; Pan \& Yuan 2012; Liu et al. 2013). Some simulations showed that the accretion flow is very dynamic and the inner radius of the flow changes with time. In addition, the flow is subject to various HD and MHD instabilities so that it is non-asymmetric. In order to avoid the complexity of solving partial differential equations, the present work is still based on the assumption of a steady and axisymmetric accretion flow. In such case, the basic equations of the neutrino-cooled accretion disk including the MC effects may refer to Section 2 of Lei et al. (2009), and the relativistic effects of the spinning BH were shown in Riffert \& Herold (1995).

For simplicity, we define the gravitational radius as $r_{\mathrm{g}} \equiv G M / c^{2}$, the dimensionless radius as $x \equiv r / r_{\mathrm{g}}$, and the dimensionless spin parameter as $a_{*} \equiv c J / G M^{2}$. The disk is assumed to be Keplerian rotating, thus the angular velocity of the flow is expressed as

$$
\Omega=\frac{c}{r} \frac{1}{\left(x^{1 / 2}+a_{*} x^{-1}\right)} .
$$

In the present work, we investigate the properties of the neutrino-cooled disk with magnetic field lines connecting the $\mathrm{BH}$ with the inner disk. Such a MC process may have

substantial effects on the energy and angular momentum balance of the disk (Li \& Paczyński 2000; Li 2002; Wang et al. 2002; Janiuk \& Yuan 2010; Kovács et al. 2011). In addition, Uzdensky (2005) showed that the inner part of the disk is magnetically coupled to the BH, 
but the magnetic field cannot be stable in the outer region. Here, we follow the assumptions of Wang et al. (2003) that the MC process is constrained by a critical polar angle $\theta_{0}$, and the magnetic field varies as a power law with the disk radius. The magnetic torque exerted to the disk from the $\mathrm{BH}$ horizon can be expressed as

$$
T_{\mathrm{MC}}=4 a_{*}\left(1+\sqrt{1-a_{*}^{2}}\right) T_{0} \int_{\theta_{0}}^{\pi / 2} \frac{(1-\beta) \sin ^{3} \theta}{2-\left(1-\sqrt{1-a_{*}^{2}}\right) \sin ^{2} \theta} d \theta
$$

where $\theta_{0}$ is a critical polar angle. In the scenario (e.g., Wang et al. 2002, Figure 1), the MC process exists in the range $\theta_{0}<\theta<\pi / 2$. On the contrary, for the space with $0<\theta<\theta_{0}$, the BZ process may occur and therefore some accreted materials may be pushed away, particularly in the inner region of the disk. In the present study, we will focus on the MC process and a constant accretion rate is assumed for simplicity. The critical angle is calculated by

$$
\cos \theta_{0}=\int_{1}^{\xi_{\text {out }}} \frac{\xi^{1-n} \chi_{\mathrm{ms}}^{2} \sqrt{1+a_{*}^{2} \chi_{\mathrm{ms}}^{-4} \xi^{-2}+2 a_{*}^{2} \chi_{\mathrm{ms}}^{-6} \xi^{-3}}}{2 \sqrt{\left(1+a_{*}^{2} \chi_{\mathrm{ms}}^{-4}+2 a_{*}^{2} \chi_{\mathrm{ms}}^{-6}\right)\left(1-2 \chi_{\mathrm{ms}}^{-2} \xi^{-1}+a_{*}^{2} \chi_{\mathrm{ms}}^{-4} \xi^{-2}\right)}} d \xi,
$$

where $\xi=r / r_{\mathrm{ms}}, \chi_{\mathrm{ms}}=\sqrt{r_{\mathrm{ms}} / r_{\mathrm{g}}}, \xi_{\text {out }}=r_{\text {out }} / r_{\mathrm{ms}}$, and $T_{0}=3.26 \times 10^{45}\left(B_{\mathrm{H}} / 10^{15} \mathrm{G}\right)^{2}\left(M / M_{\odot}\right)^{3}$ $\mathrm{g} \mathrm{cm}^{2} \mathrm{~s}^{-2} . \beta=\Omega / \Omega_{\mathrm{H}}$ is the ratio of the angular velocity of the disk (Eq. (1)) to the angular velocity at the horizon, where $\Omega_{\mathrm{H}}=\left(c a_{*} / 2 r_{\mathrm{g}}\right) /\left(1+\sqrt{1-a_{*}^{2}}\right)$. In addition, under the equipartition assumption (e.g., McKinney 2005; Lei et al. 2009), the magnetic field strength at the horizon can be estimated as $B_{\mathrm{H}}^{2}=8 \pi c \dot{M} / r_{\mathrm{g}}^{2}$.

The energy equation is written as

$$
Q_{\mathrm{vis}}^{+}=Q_{G}^{+}+Q_{\mathrm{MC}}^{+}=Q_{\mathrm{adv}}^{-}+Q_{\nu}^{-}
$$

where $Q_{\text {vis }}^{+}$is the viscous heating rate, including the contributions of the gravitational potential $Q_{G}^{+}$and the MC process $Q_{\mathrm{MC}}^{+}=-T_{\mathrm{MC}} /(4 \pi r) \cdot d \Omega / d r$. The quantities $Q_{\mathrm{adv}}^{-}$and $Q_{\nu}^{-}$ are respectively the advective cooling rate and the neutrino cooling rate. Here, we neglect the radiation of photons since they are trapped in the disk. The neutrino cooling generally consists of the four processes: the electron-positron pair annihilation, the bremsstrahlung emission of nucleons, the plasmon decay, and the Urca process (e.g., Liu et al. 2007). In addition, for the equation of state, the total pressure consists of five terms, i.e., the gas pressure, the radiation pressure, the degeneracy pressure, the neutrino pressure, and the magnetic pressure. The detailed description of the neutrino cooling and the pressure can be found in some previous papers (e.g., Popham et al. 1999; Gu et al. 2006; Janiuk \& Yuan 2010). For the term of magnetic pressure, following Lei et al. (2009), the ratio of magnetic pressure to total pressure is assumed to be 0.1 . 


\section{Numerical results}

\subsection{Disk structure}

In our calculations we fix $M=3 M_{\odot}, \alpha=0.1, a_{*}=0.9, n=3$, and $r_{\text {out }}=100 r_{\mathrm{g}}$. The numerical results of structure and radiation are shown in Figure 1, where the solid lines and the dashed lines represent the solutions with and without the MC process, respectively. We choose three accretion rates, i.e., $\dot{M}=0.005,0.05$, and $0.5 M_{\odot} \mathrm{s}^{-1}$ for the study. Figures $1 \mathrm{a}$, $1 \mathrm{~b}$, and 1c show respectively the radial profiles of the mass density, the temperature, and the neutrino cooling efficiency defined as the ratio of the neutrino cooling rate to the viscous heating rate $Q_{\nu}^{-} / Q_{\mathrm{vis}}^{+}$.

It is seen that, for the outer region with $r \gtrsim 20 r_{\mathrm{g}}$, the solid and dashed lines are identical to each other, which implies that the $\mathrm{MC}$ effects in this region are negligible. On the contrary, for the inner region with $r \lesssim 20 r_{\mathrm{g}}$, the solid lines and the dashed lines are apparently separate, which indicates that the $\mathrm{MC}$ effects are substantial. For the inner region, Figure 1a shows that the density with MC is obviously larger than that without MC. The physical understanding is as follows. The MC process can transfer angular momentum from the $\mathrm{BH}$ to the inner disk. As a consequence, the additional transferred angular momentum will work as a barrier to prevent the flow from radial acceleration, and therefore the accreted matter will accumulate in this region and the mass density will significantly increase. On the other hand, for $\dot{M}=0.5 M_{\odot} \mathrm{s}^{-1}$, it is shown by Figure 1a that the solid line drops

inwards even faster than the dashed line. The reason is that, for large $\dot{M}$, the disk will become optically thick to neutrinos. Thus, most of the generated neutrinos are trapped in the disk instead of escaping away. The total pressure, which includes the neutrino pressure, will therefore increase significantly and the disk will probably become geometrically thick. Accordingly, the mass density will drop sharply due to the increased vertical height and radial velocity. The neutrino trapping can be also indicated by Figure 1c, where the solid line steeply drops inwards for $\dot{M}=0.5 M_{\odot} \mathrm{s}^{-1}$, indicating that most neutrinos are trapped in the disk rather than being radiated.

\subsection{Annihilation luminosity}

In the scenario of hyper-accretion disks, the GRBs are powered by the neutrino and anti-neutrino annihilation above the surface of disks. Since the X-ray flares and the gammaray emission have the same power origin, the flares may still be powered by the neutrino annihilation. The disk luminosity is calculated from the marginal stable orbit $r_{\mathrm{ms}}$ to the 
outer boundary $r_{\text {out }}$. The neutrino luminosity from the disk is calculated as

$$
L_{\nu}=4 \pi \int_{r_{\mathrm{ms}}}^{r_{\mathrm{out}}} r Q_{\nu}^{-} d r .
$$

The total annihilation luminosity is calculated by the integration over the whole space outside the disk, following the method in previous works (e.g., Ruffert et al. 1997; Popham et al. 1999).

Figure 2 shows the variations of the neutrino luminosity $L_{\nu}\left(L_{\nu}^{\prime}\right)$ and the annihilation

luminosity $L_{\nu \bar{\nu}}\left(L_{\nu \bar{\nu}}^{\prime}\right)$ with the mass accretion rate $\dot{M}$. Similar to Figure 1 , the solid lines and the dashed lines represent the solutions with and without the MC process, respectively. The upper solid (dashed) line corresponds to $L_{\nu}\left(L_{\nu}^{\prime}\right)$, and the lower solid (dashed) line corresponds to $L_{\nu \bar{\nu}}\left(L_{\nu \bar{\nu}}^{\prime}\right)$. It is seen that, both the neutrino luminosity and the annihilation luminosity with the MC process are significantly larger than those without the MC process. In particular for relatively low accretion rates such as $\dot{M} \sim 0.01 M_{\odot} \mathrm{s}^{-1}, L_{\nu \bar{\nu}}$ is larger than $L_{\nu \bar{\nu}}^{\prime}$ by up to four orders of magnitude. The physical reason is that, in addition to the gravitational energy, the $\mathrm{MC}$ process can efficiently extract the $\mathrm{BH}$ rotational energy into neutrino radiation. Moreover, the apparent difference between the lower solid line and the lower dashed line implies that it is quite possible for a remnant low-mass disk to power X-ray flares.

\subsection{Efficiency of $\mathrm{BH}$ rotational energy extraction}

We compare the efficiency of BH rotational energy extraction between the MC process and the BZ mechanism. The former efficiency is defined as

$$
\eta_{\mathrm{MC}}=\frac{\int_{r_{\mathrm{ms}}}^{r_{\mathrm{out}}} 4 \pi r Q_{\mathrm{MC}}^{+} d r}{\dot{M} c^{2}} .
$$

For the BZ mechanism, the magnetic field lines, which are dragged in by the accretion disk, accumulate around the $\mathrm{BH}$ horizon and then get twisted by the $\mathrm{BH}$ space-time, which enable the extraction of BH rotational energy. The efficiency of the BZ process was discussed in Tchekhovskoy et al. (2011),

$$
\eta_{\mathrm{BZ}}=\frac{\kappa}{4 \pi c}\left(\frac{\Omega_{\mathrm{H}} r_{g}}{c}\right)^{2} \Phi_{0}^{2} f\left(\Omega_{\mathrm{H}}\right),
$$

where $\Phi_{0}=\Phi_{\mathrm{BH}} /\left(\dot{M} c r_{g}^{2}\right)^{1 / 2}$ is the dimensionless magnetic flux threading the $\mathrm{BH}, \kappa$ is a numerical constant related to the magnetic field geometry (we adopt $\kappa=0.044$ here), and 
$f\left(\Omega_{\mathrm{H}}\right)$ is set to be 1 . The above equation shows that the BZ efficiency is relevant to the dimensionless magnetic flux $\Phi_{0}$. The value of $\Phi_{0}$, however, is quite uncertain and may be related to the accretion rate. It can be regarded as the ability for the accretion disk to drag magnetic fields into the $\mathrm{BH}$ horizon. Recent simulations show that $\Phi_{0}$ can be as large as several tens and the disk can be depicted as a magnetic arrest disk. At a high $\Phi_{0}$, a large amount of magnetic flux is transported to the center and the efficiency can be larger than 1 . Here we simply assume a constant magnetic flux, $\Phi_{0}=50$, which is close to the simulation result $\Phi_{0} \approx 47$ (Tchekhovskoy et al. 2011).

Equations (6) implies that $\eta_{\mathrm{MC}}$ is independent of the accretion rate $\dot{M}$. The variation of $\eta_{\mathrm{MC}}$ and $\eta_{\mathrm{BZ}}$ with the spin parameter $a_{*}$ is shown in Figure 3 . It is seen that, for $a_{*} \gtrsim 0.5, \eta_{\mathrm{MC}}$ can be significantly larger than the efficiency of normal accretion process $0.06 \lesssim \eta \lesssim 0.42$, and can be even larger than 1 for extremely spinning BH. Such a result indicates that the MC process is quite an efficient mechanism to extract the $\mathrm{BH}$ rotational energy. The efficiency $\eta_{\mathrm{MC}}$ decreases sharply with decreasing $a_{*}$ for $a_{*} \lesssim 0.4$. The reason is that the angular velocity of BH will be less than that of the disk at ISCO for $a_{*} \lesssim 0.36$, and therefore the MC process will become weak for BH spin below this critical value. It is also seen that, for $a_{*} \gtrsim 0.4$, $\eta_{\mathrm{MC}}$ is several times larger than $\eta_{\mathrm{BZ}}$, whereas for $a_{*} \lesssim 0.3$, $\eta_{\mathrm{BZ}}$ is significantly larger than $\eta_{\mathrm{MC}}$ due to a sharp decrease of $\eta_{\mathrm{MC}}$ with decreasing $a_{*}$. In other words, for fast spinning BH systems, MC may be more powerful than BZ on the extraction of rotational energy, whereas for slow spinning BH systems, BZ is likely to be more powerful.

Another different effect between $\mathrm{MC}$ and $\mathrm{BZ}$ is that, the BZ process can directly transfer the rotational energy into the jet, whereas the $\mathrm{MC}$ process can only transfer the rotational energy into the disk, and the jet power is also related to another process, i.e., neutrino annihilation. Thus, for the efficiency of powering the jet, the BZ process will probably be much more efficient. For a comparison, the dotted line shows the efficiency of radiation due to neutrino annihilation $\eta_{\nu \bar{\nu}}\left(\equiv L_{\nu \bar{\nu}} / \dot{M} c^{2}\right)$ for a typical accretion rate $\dot{M}=0.05 M_{\odot} \mathrm{s}^{-1}$. It is seen that $\eta_{\mathrm{BZ}}$ is significantly larger than $\eta_{\nu \bar{\nu}}$ for any $a_{*}$. Thus, the BZ process is more efficient to power a jet than the MC process. It is also possible for the BZ process to work as the central engine to power the X-ray flares.

\section{Comparison with observations}

In order to compare our numerical results with the observations of X-ray flares, we compile a sample of 21 GRBs with 43 flares as shown in Table 1, which includes all the flares with available redshift in Table 1 of Chincarini et al. (2010). The width of the flares is calculated in the rest frame: $w_{\text {res }}=w /(1+z)$, where $z$ is the redshift, $w$ is the observed 
width, and $w_{\text {res }}$ is the width in the rest frame. The isotropic energy of a flare $E_{\text {flare }}$ can be estimated from the fluence $S$ : $E_{\text {flare }}=4 \pi D_{l}^{2} S /(1+z)$, where $D_{l}$ is the luminosity distance. Thus, the average, isotropic luminosity can be obtained as $L_{\text {iso }}=E_{\text {flare }} / w_{\text {res }}$.

A comparison of our numerical results with the observations is shown in Figure 4, which includes all the flares in Table 1. Similar to Figures 1 and 2, the solid lines represent the results with $\mathrm{MC}$, whereas the dashed lines represent the results without MC. The upper and lower solid (dashed) lines correspond to the disk mass $M_{\text {disk }}=0.5 M_{\odot}$ and $0.05 M_{\odot}$, respectively. The four theoretical lines are calculated by $w_{\text {res }}=M_{\text {disk }} / \dot{M}$ together with the relationship between $\dot{M}$ and $L_{\nu \bar{\nu}}$ obtained in Section 3.

Figure 4 shows that most of the flares locate above the upper dashed line, which means that for the case without MC, it will generally require a remnant disk mass $M_{\text {disk }}$ significantly larger than $0.5 M_{\odot}$, which may be unpractical. However, it is seen that most of the flares exist between the two solid lines, which indicates that if MC works, a reasonable remnant disk with $0.05 M_{\odot} \lesssim M_{\text {disk }} \lesssim 0.5 M_{\odot}$ is able to power nearly all the plotted flares. As mentioned in Section 1, taking the second flare of GRB 070318 as an example, the model without $\mathrm{MC}$ requires $M_{\mathrm{disk}} \approx 4.8 M_{\odot}$. On the contrary, if the $\mathrm{MC}$ effects are taken into account, the luminosity of $2.96 \times 10^{48} \mathrm{erg} \mathrm{s}^{-1}$ corresponds to $\dot{M} \approx 0.0032 M_{\odot} \mathrm{s}^{-1}$ (according to our numerical calculations shown by the lower solid line in Figure 2). Therefore, the rest frame duration $80.6 \mathrm{~s}$ only requires $M_{\text {disk }} \approx 0.26 M_{\odot}$ for powering this flare.

\section{Conclusions and discussion}

In this paper, we have studied the neutrino-cooled disks by taking into account the MC process between the central $\mathrm{BH}$ and the inner disk. We have shown that, for mass accretion rates around $0.001 \sim 0.1 M_{\odot} \mathrm{s}^{-1}$, the luminosity of neutrino annihilation can be enhanced by up to four orders of magnitude due to the MC effects. As a consequence, the remnant disk with $M_{\text {disk }} \lesssim 0.5 M_{\odot}$ may power most of the observed X-ray flares with the rest frame duration less than 100 seconds.

We would point out that, for a few X-ray flares with extremely long duration, the neutrino-cooled disk cannot work as the central engine, even though $\mathrm{MC}$ is included. For example, GRB 050724 has three flares with a redshift of 0.258 . The third flare of this source has a rest frame width of $3.1 \times 10^{5}$ seconds and the luminosity $6.7 \times 10^{43} \mathrm{erg} \mathrm{s}^{-1}$. According to our numerical results in Figure 2, the accretion rate should be around $1.7 \times 10^{-4} M_{\odot} \mathrm{s}^{-1}$ for the case including MC. Thus, it requires a remnant disk $M_{\text {disk }}>50 M_{\odot}$, which is obviously unphysical. On the other hand, X-ray flares with peak time less than and larger than 1000 
s may have different origin (Margutti et al. 2011). The mechanism for powering the flares with peak time larger than $1000 \mathrm{~s}$ is worthy for further studies, but is beyond the scope of the present paper.

Another issue we would like to stress is related to the configuration of the large-scale magnetic field in accretion disks. Although the MC process has been studied by quite a few previous works, such type of magnetic fields, however, has not been found in MHD simulations. Thus, it remains unclear whether the MC process can occur between the inner disk and the central BH. On the other hand, some simulations showed that the BZ mechanism can be a solution to the GRB's central engine (e.g., Tchekhovskoy et al. 2008). In the scenario of the Poynting flux-dominated jet, the efficiency of extracting the BH rotational energy mainly depends on the magnetic flux being dragged in (Tchekhovskoy et al. 2011; McKinney et al. 2012). The theoretical analysis showed that it requires a geometrically thick disk to transport a large mount flux into the center (Lubow et al. 1994; Rothstein \& Lovelace 2008; Beckwith et al. 2009; Cao 2011). Simulations also confirmed that a thick disk can efficiently transport magnetic flux (McKinney et al. 2012). For a neutrino-cooled disk, neutrinos play a vital role to release the dissipation heat and the disk is likely to be geometrically thin (e.g., Shibata et al. 2007). Then, for the Poynting flux-dominated jet, it remains a problem whether the accretion flow can accumulate adequate magnetic fields to the inner region to power the jet.

In this work, the flow is assumed to be steady and the mass accretion rate is a free parameter. In other words, for a given accretion rate, we will obtain a corresponding solution. On the other hand, the simulations of Tchekhovskoy et al. (2011) found a correlated variation between the accretion rate and the magnetic flux $\Phi_{\mathrm{BH}}$. In this spirit, a varying strength of MC process may also have effects on the variation of accretion rate. Such a study requires further time-dependent calculations.

We thank Raffaella Margutti, Shujin Hou, Da-Bin Lin, and Mou-Yuan Sun for beneficial discussions, and the referee for helpful suggestions. This work was supported by the National Natural Science Foundation of China under grants 11073015, 11103015, 11222328, and 11233006 .

\section{REFERENCES}

Beckwith, K., Hawley, J. F., \& Krolik, J. H. 2009, ApJ, 707, 428 
Bernardini, M. G., Margutti, R., Chincarini, G., Guidorzi, C., \& Mao, J. 2011, A\&A, 526, A27

Blandford, R. D., \& Znajek, R. L. 1977, MNRAS, 179, 433

Cao, X. 2011, ApJ, 737, 94

Chen, W.-X., \& Beloborodov, A. M. 2007, ApJ, 657, 383

Chincarini, G., Moretti, A., Romano, P., et al. 2007, ApJ, 671, 1903

Chincarini, G., Mao, J., Margutti, R., et al. 2010, MNRAS, 406, 2113

Dai, Z. G., Wang, X. Y., Wu, X. F., \& Zhang, B. 2006, Science, 311, 1127

Di Matteo, T., Perna, R., \& Narayan, R. 2002, ApJ, 579, 706

Falcone, A. D., Morris, D., Racusin, J., et al. 2007, ApJ, 671, 1921

Gehrels, N., Ramirez-Ruiz, E., \& Fox, D. B. 2009, ARA\&A, 47, 567

Gu, W.-M., Liu, T., \& Lu, J.-F. 2006, ApJ, 643, L87

Janiuk, A., \& Yuan, Y.-F. 2010, A\&A, 509, A55

King, A. R., et al. 2005, ApJ, 630, L113

Kovács, Z., Gergely, L., \& Biermann, P. L. 2011, MNRAS, 416, 991

Lazzati, D., Blackwell, C. H., Morsony, B. J., \& Begelman, M. C. 2011, MNRAS, 411, L16

Lazzati, D., Perna, R., \& Begelman, M. C. 2008, MNRAS, 388, L15

Lee, W. H., Ramirez-Ruiz, E., \& López-Cámara D. 2009, ApJ, 699, L93

Lei, W. H., Wang, D. X., Zhang, L., et al. 2009, ApJ, 700, 1970

Li, L.-X. 2002, ApJ, 567, 463

Li, L.-X., \& Paczyński, B. 2000, ApJ, 534, L197

Liu, T., Gu, W.-M., Xue, L., \& Lu, J.-F. 2007, ApJ, 661, 1025

Liu, T., Xue, L., Gu, W.-M., \& Lu, J.-F. 2013, ApJ, 762, 102

Lubow, S. H., Papaloizou, J. C. B., \& Pringle, J. E. 1994, MNRAS, 267, 235 
Margutti, R., Bernardini, G., Barniol Duran, R., et al. 2011, MNRAS, 410, 1064

McKinney, J. C. 2005, ApJ, 630, L5

McKinney, J. C., \& Gammie, C. F. 2004, ApJ, 611, 977

McKinney, J. C., Tchekhovskoy, A., \& Blandford, R. D. 2012, MNRAS, 423, 3083

Mészáros, P. 2006, Reports on Progress in Physics, 69, 2259

Pan, Z., \& Yuan, Y.-F. 2012, ApJ, 759, 82

Pannarale, F., Tonita, A., \& Rezzolla, L. 2011, ApJ, 727, 95

Perna, R., Armitage, P. J., \& Zhang, B. 2006, ApJ, 636, L29

Popham, R., Woosley, S. E., \& Fryer, C. 1999, ApJ, 518, 356

Proga, D., \& Zhang, B. 2006, MNRAS, 370, L61

Riffert, H., \& Herold, H. 1995, ApJ, 450, 508

Romano, P., Moretti, A., Banat, P. L., et al. 2006, A\&A, 450, 59

Rothstein, D. M., \& Lovelace, R. V. E. 2008, ApJ, 677, 1221

Ruffert, M., Janka, H. -T., Takahashi, K., \& Schaefer, G. 1997, A\&A, 319, 122

Shibata, M., Sekiguchi, Y.-I., \& Takahashi, R. 2007, Progress of Theoretical Physics, 118, 257

Shibata, M., \& Taniguchi, K. 2008, Phys. Rev. D, 77, 084015

Tchekhovskoy, A., McKinney, J. C., \& Narayan, R. 2008, MNRAS, 388, 551

Tchekhovskoy, A., Narayan, R., \& McKinney, J. C. 2011, MNRAS, 418, L79

Uzdensky, D. A. 2005, ApJ, 620, 889

Wang, D. X., Xiao, K., \& Lei, W. H. 2002, MNRAS, 335, 655

Wang, D. X., Lei, W. H., \& Ma, R. Y. 2003, MNRAS, 342, 851

Yuan, F., \& Zhang, B. 2012, ApJ, 757, 56 

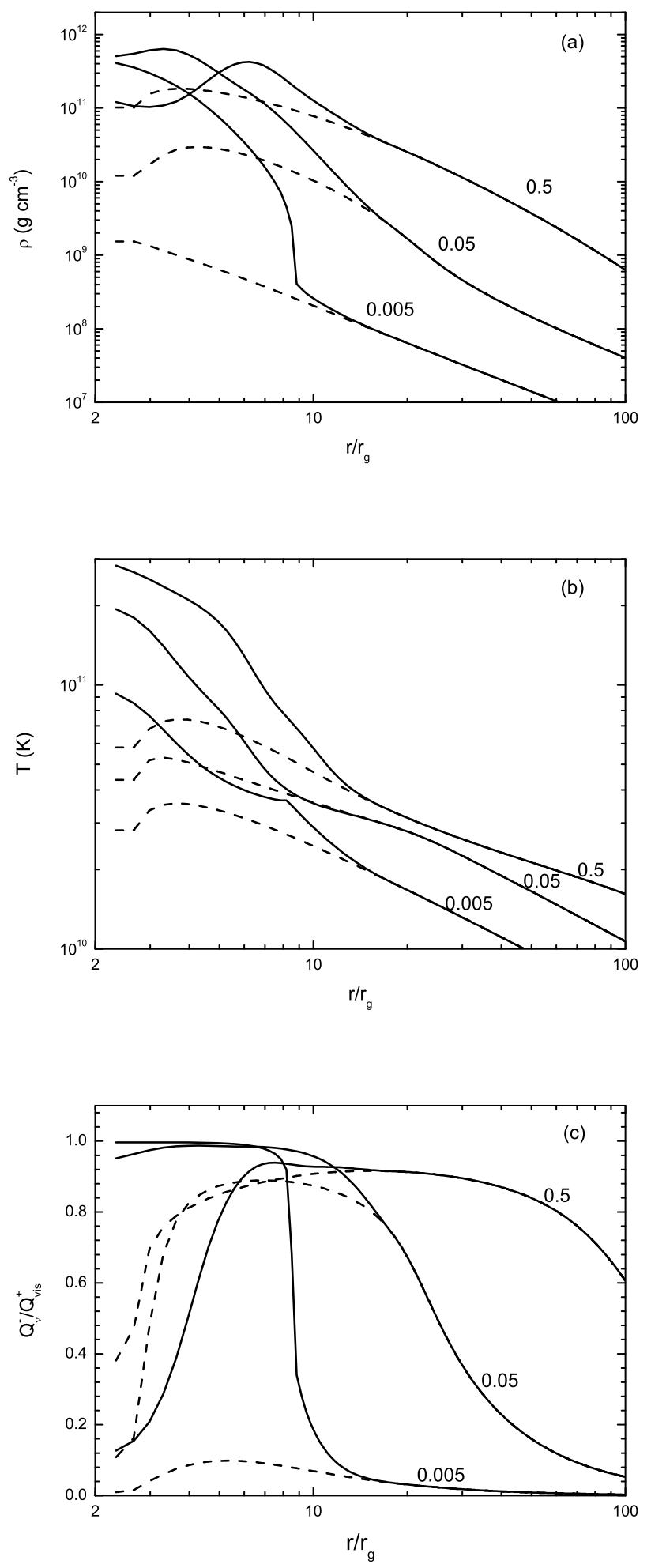

Fig. 1. - Radial profiles of the density $\rho$, the temperature $T$, and the ratio of the neutrino cooling to the viscous heating $Q_{\nu}^{-} / Q_{\text {vis }}^{+}$for $\dot{M}=0.005,0.05,0.5 M_{\odot} \mathrm{s}^{-1}$. The solid lines and the dashed lines represent the solutions with and without the MC process, respectively. 


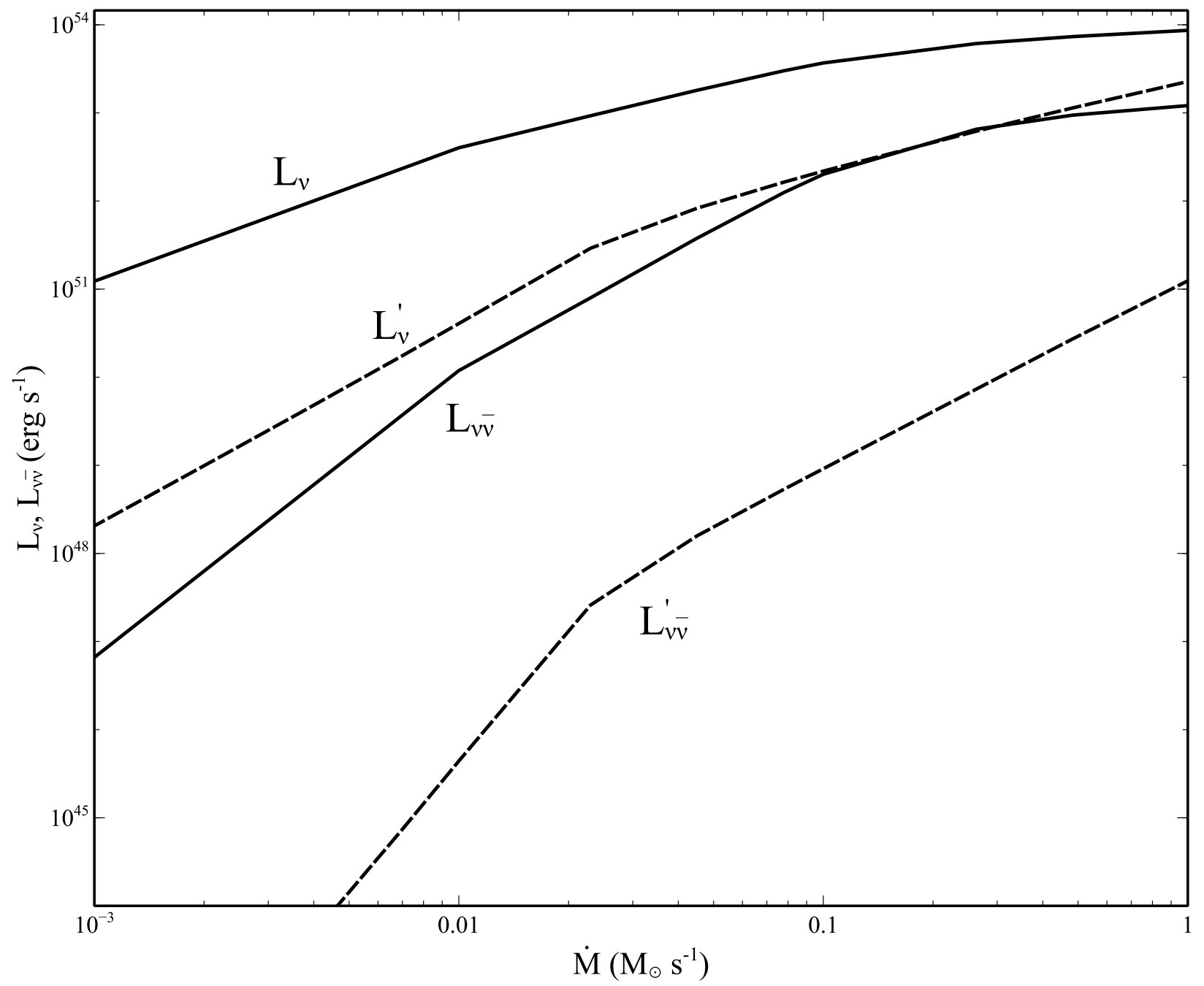

Fig. 2.- Variations of the neutrino luminosity $L_{\nu}\left(L_{\nu}^{\prime}\right)$ and the annihilation luminosity $L_{\nu \bar{\nu}}$ $\left(L_{\nu \bar{\nu}}^{\prime}\right)$ with the mass accretion rate. The upper and lower solid lines correspond to $L_{\nu}$ and $L_{\nu \bar{\nu}}$ with the MC process, respectively, whereas the upper and lower dashed lines correspond to $L_{\nu}^{\prime}$ and $L_{\nu \bar{\nu}}^{\prime}$ without the MC process, respectively. 


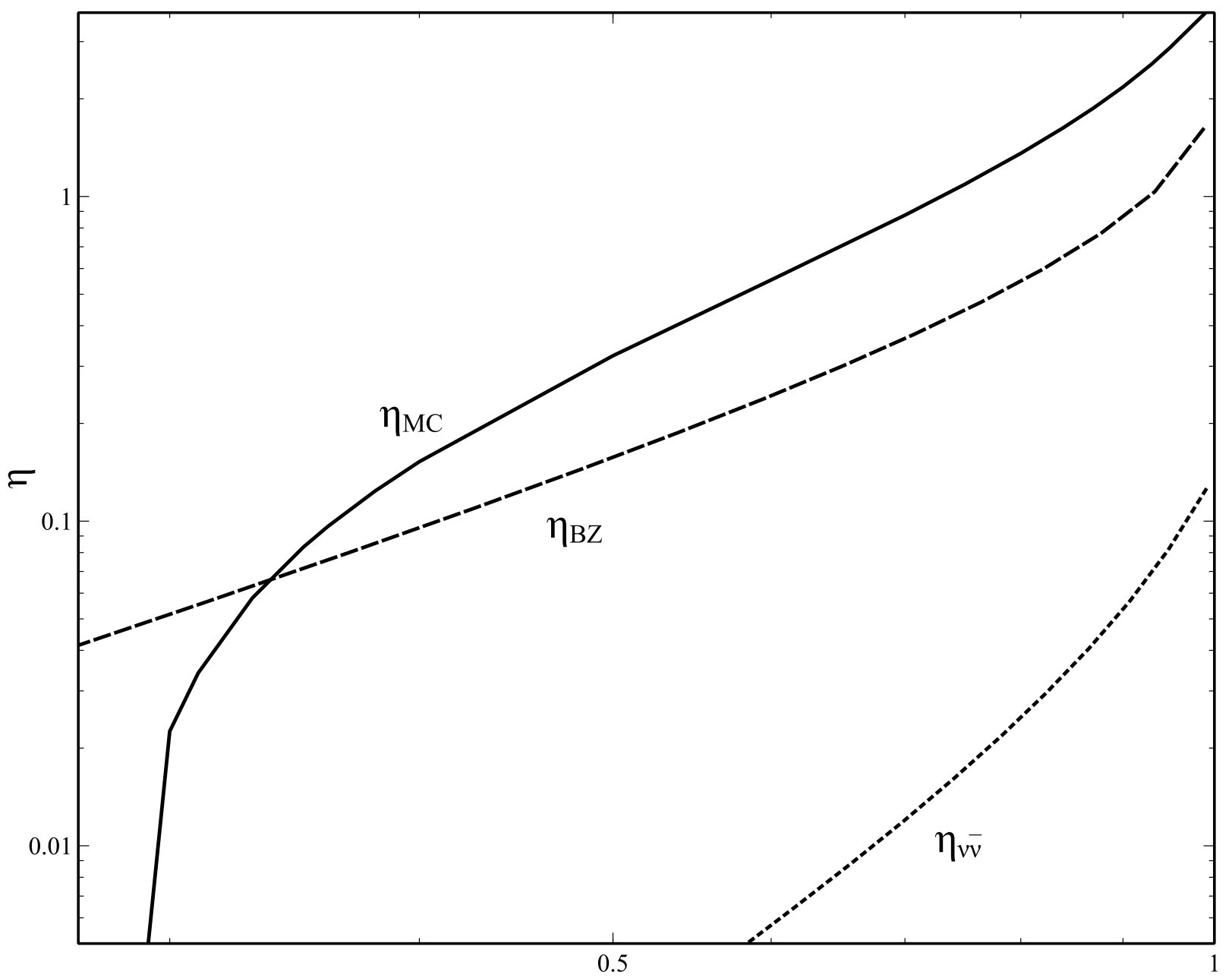

$a_{\text {* }}$

Fig. 3.- Variation of the efficiency $\eta$ with the spin parameter $a_{*}$. The solid line shows the efficiency of the energy transfer from the rotating $\mathrm{BH}$ to the disk by the MC process. For a comparison, the dashed line shows the efficiency of the energy extraction by the BZ mechanism with $\Phi_{0}=50$. The dotted line shows the efficiency of radiation due to neutrino annihilation for a typical accretion rate $\dot{M}=0.05 M_{\odot} \mathrm{s}^{-1}$. 


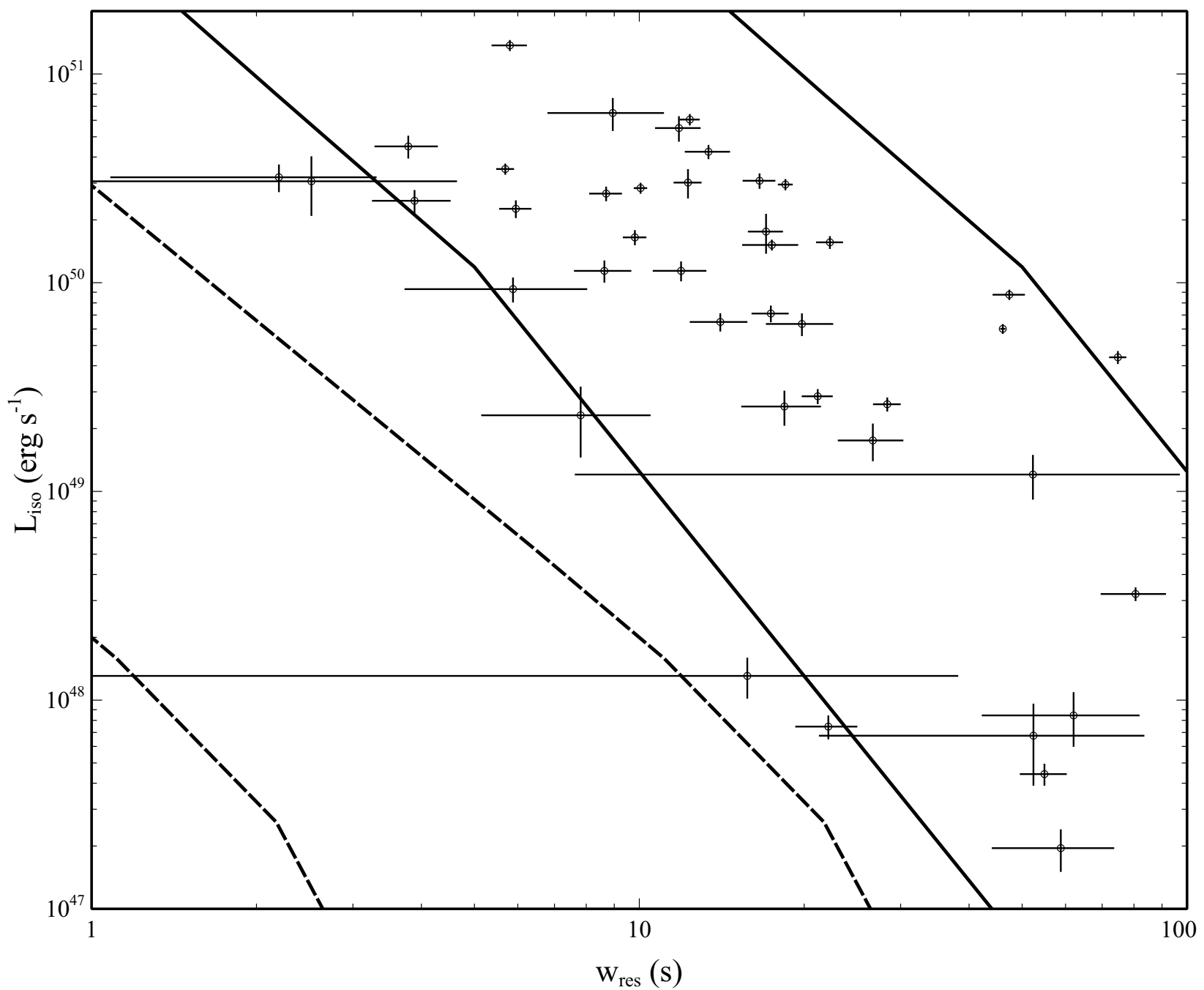

Fig. 4.- A comparison of our numerical results with the observations in the $L_{\text {iso }}-w_{\text {res }}$ diagram. The upper and lower solid lines correspond to the remnant disk mass $M_{\text {disk }}=$ $0.5 M_{\odot}$ and $0.05 M_{\odot}$ with the $\mathrm{MC}$ process, respectively, whereas the upper and lower dashed lines correspond to $M_{\text {disk }}=0.5 M_{\odot}$ and $0.05 M_{\odot}$ without the MC process, respectively. The data for the 43 flares are shown in Table 1. 
Table 1. Observed flares with available redshift

\begin{tabular}{cccc}
\hline \hline GRB & $\mathrm{z}$ & $L_{\text {iso }}\left(10^{50} \mathrm{erg} \mathrm{s}^{-1}\right)$ & $w_{\text {res }}(\mathrm{s})$ \\
\hline 050730 & 3.967 & $1.0210 \pm 0.4929$ & $8.6370 \pm 1.0268$ \\
050730 & 3.967 & $1.3644 \pm 0.3684$ & $22.2871 \pm 1.2684$ \\
050730 & 3.967 & $0.5679 \pm 0.2609$ & $19.8108 \pm 2.7783$ \\
050908 & 3.344 & $0.1648 \pm 0.0520$ & $26.6805 \pm 3.6602$ \\
060115 & 3.53 & $0.2332 \pm 0.0960$ & $18.4106 \pm 3.0464$ \\
060210 & 3.91 & $5.5296 \pm 0.9617$ & $12.3625 \pm 0.5295$ \\
060210 & 3.91 & $2.9090 \pm 0.5818$ & $12.2607 \pm 0.7128$ \\
060418 & 1.489 & $2.6893 \pm 0.2241$ & $10.0442 \pm 0.2812$ \\
060512 & 0.4428 & $0.0041 \pm 0.0010$ & $54.8933 \pm 5.4062$ \\
060526 & 3.221 & $12.0001 \pm 14.6252$ & $5.8043 \pm 0.4264$ \\
060526 & 3.221 & $6.0765 \pm 2.6736$ & $8.9552 \pm 2.1559$ \\
060526 & 3.221 & $4.9712 \pm 1.1600$ & $11.8218 \pm 1.1372$ \\
060526 & 3.221 & $1.6612 \pm 0.4345$ & $17.0339 \pm 1.2556$ \\
060604 & 2.68 & $2.3969 \pm 0.6453$ & $8.6957 \pm 0.5978$ \\
060604 & 2.68 & $2.1283 \pm 0.3502$ & $5.9511 \pm 0.4076$ \\
$060607 \mathrm{~A}$ & 3.082 & $2.2870 \pm 0.7277$ & $3.8951 \pm 0.6369$ \\
$060607 \mathrm{~A}$ & 3.082 & $2.6306 \pm 0.3617$ & $18.4713 \pm 0.5879$ \\
060707 & 3.425 & $0.2153 \pm 0.1846$ & $7.8192 \pm 2.6667$ \\
060714 & 2.711 & $4.0362 \pm 0.5259$ & $13.3657 \pm 1.2665$ \\
060714 & 2.711 & $2.8113 \pm 0.9618$ & $2.2096 \pm 1.1048$ \\
060714 & 2.711 & $4.1165 \pm 1.0400$ & $3.7726 \pm 0.5120$ \\
060714 & 2.711 & $3.1626 \pm 0.5750$ & $5.6858 \pm 0.2156$ \\
060729 & 0.54 & $0.2463 \pm 0.0265$ & $28.3766 \pm 1.6234$ \\
060814 & 0.84 & $0.2704 \pm 0.0419$ & $21.1957 \pm 1.3587$ \\
$060904 \mathrm{~B}$ & 0.703 & $0.5598 \pm 0.0336$ & $46.0951 \pm 0.5285$ \\
060908 & 1.8836 & $0.0066 \pm 0.0066$ & $52.4345 \pm 31.1416$ \\
060908 & 1.8836 & $0.0070 \pm 0.0098$ & $62.0752 \pm 19.8710$ \\
070306 & 1.4959 & $0.6855 \pm 0.1143$ & $17.3885 \pm 1.3622$ \\
070318 & 0.836 & $0.0116 \pm 0.0128$ & $15.7407 \pm 22.4401$ \\
070318 & 0.836 & $0.0296 \pm 0.0043$ & $80.5556 \pm 11.0022$ \\
$070721 \mathrm{~B}$ & 3.626 & $0.6013 \pm 0.8718$ & $26.3078 \pm 63.3593$ \\
$070721 \mathrm{~B}$ & 3.626 & $2.7102 \pm 1.8763$ & $2.5292 \pm 2.1185$ \\
$070721 \mathrm{~B}$ & 3.626 & $0.8519 \pm 0.3139$ & $5.8798 \pm 2.1617$ \\
$070721 \mathrm{~B}$ & 3.626 & $0.1109 \pm 0.0907$ & $52.3130 \pm 44.6822$ \\
$070724 \mathrm{~A}$ & 0.457 & $0.0067 \pm 0.0058$ & $22.1688 \pm 2.8826$ \\
$070724 \mathrm{~A}$ & 0.457 & $0.0018 \pm 0.0009$ & $58.8195 \pm 14.8250$ \\
071031 & 2.692 & $2.7287 \pm 1.0720$ & $16.5764 \pm 1.1376$ \\
071031 & 2.692 & $1.0844 \pm 0.2575$ & $11.9177 \pm 1.3272$ \\
071031 & 2.692 & $0.6206 \pm 0.1494$ & $14.0574 \pm 1.7064$ \\
071031 & 2.692 & $0.4104 \pm 0.0410$ & $74.7833 \pm 2.7086$ \\
080210 & 2.641 & $1.4834 \pm 0.3350$ & $9.8050 \pm 0.4944$ \\
080310 & 2.42 & $0.8247 \pm 0.0654$ & $47.3684 \pm 3.2164$ \\
080310 & 2.42 & $1.3890 \pm 0.1543$ & $17.4561 \pm 2.0468$ \\
\hline & & & \\
\hline
\end{tabular}

